\title{
Constructive Approach to Three Dimensional Sklyanin Algebras
}

\section{Natalia lyudu $^{1 *}$ and Stanislav Shkarin ${ }^{2}$}

${ }^{1}$ School of Mathematics, The University of Edinburgh, The King's Buildings, Mayfield Road, Edinburgh, Scotland EH9 3JZ

${ }^{2}$ Queens's University Belfast, Department of Pure Mathematics, University Road, Belfast, BT7 1NN, UK

\begin{abstract}
A three dimensional Sklyanin is the quadratic algebra over a field $\mathbb{k}$ with 3 generators $x ; y ; z$ given by 3 relations $x y-a y x-s z z=0, y z-a z y-s x x=0$ and $z x-a x z-s y y=0$, where $a, s \in \mathbb{K}$. A generalized Sklyanin algebra is the algebra given by relations $x y-a_{1} y x-s_{1} z z=0, y z-a_{2} z y-s_{2} x x=0$ and $z x-a_{3} x z-s_{3} y y=0$, where $a_{i}, s_{i} \in \mathbb{K}$. In this paper we announce the following results; the complete proofs will appear elsewhere. We determine explicitly the parameters for which these algebras has the same Hilbert series as the algebra of commutative polynomials in 3 indeterminates as well as when these algebras are Koszul and PBW, using constructive combinatorial methods. These provide new direct proofs of results established first by Artin, Tate, and Van Den Bergh.
\end{abstract}

Keywords: Quadratic algebras; Koszul algebras; Hilbert series; Grobner bases; PBWalgebras; PHSalgebras

\section{Introduction}

It is well-known that algebras arising in string theory, from the geometry of Calabi-Yau manifolds, i.e. various versions of CalabiYau algebras, enjoy the potentiality-like properties. This in essence comes from the symplectic structure on the manifold. The notion of noncommutative potential was first introduced by Kontsevich in [1]. Let $F=\mathbb{C}\left\langle x_{1}, \ldots \mathrm{x}_{\mathrm{n}}\right\rangle$, then the quotient vector space $F c y c=F /[F, F]$ has a simple basis labeled by cyclic words in the alphabet $x_{1} \ldots x_{n}$. For each $j$ $=1, \ldots, n$ in $[1]$ was introduced a linear map $\frac{\delta}{\delta_{x}}: F_{c y c} \rightarrow F: \Phi \mapsto \frac{\delta \Phi}{\delta_{x}}$.

$$
\frac{\delta \Phi}{\delta_{x_{j}}}=\sum_{s i_{s}=j} x_{i_{s}}+1 x_{i_{s}}+2 \ldots \mathrm{x}_{\mathrm{i}_{\mathrm{r}}} x_{i_{1}} x_{i_{2}} \ldots x_{i_{s}}-1
$$

So, for any element $\Phi \in F_{c y c}$, which is called potential, one can define a collection of elements $\frac{\delta \Phi}{\delta_{x_{i}}}, i=1, \ldots, n$ an algebra which has a
presentation:

$$
u=\mathbb{C}\left\langle x_{1}, \ldots, x_{n}\right\rangle /\left\{\frac{\delta \Phi}{\delta x_{i}}\right\} i=1, \ldots n
$$

is called a potential algebra. This can be generalized to super potential algebras. It is known for 3-dimensional Calabi-Yau that they are always derived from a super potential. But not all super potential algebras are Calabi-Yau. This question was studied in details in [2-5] the conditions on potential which ensure CY has been studied. The most general counterpart of potentiality and its relation to CY (in one of possible definitions) considered in [6]. The simplest example of potential algebras are commutative polynomials. Another important example, which have been studied thoroughly [7] are Sklyanin algebras. We are aiming here to demonstrate, that such properties of these algebras as PBW, PHS, Kosulity could be obtained by constructive, purely combinatorial and algebraic methods, avoiding the power of geometry demonstrated in [ATV1, ATV2] and later papers continuing this line.

Throughout this paper $\mathbb{k}$ is an arbitrary field, $B$ is a graded algebra, and the symbol $B m$ stands for the $m^{\text {th }}$ graded component of algebra $B$. If $V$ is an $n$-dimensional vector space over $\mathbb{k}$, then $F=F(V)$ is the tensor algebra of $V$. For any choice of a basis $x_{1, \ldots}, x_{n}$ in $V, F$ is naturally identified with the free $\mathbb{k}$ algebra with the generators $x_{1}, \ldots, x_{n}$. For subsets $P_{1}, \ldots, P_{k}$ of an algebra $B, P_{1}, \ldots, P_{k}$ stands for the linear span of all products $p_{1} \ldots p_{k}$ with $p_{j} \in P_{j}$. We consider a degree grading on the free algebra $F$ : the $m^{\text {th }}$ graded component of $F$ is $V^{m}$.

If $R$ is a subspace of the $n^{2}$-dimensional space $V \otimes V$, then the quotient of $F$ by the ideal $I$ generated by $R$ is called a quadratic algebra and denoted $A(V, R)$. For any choice of bases $x_{1}, \ldots, x_{n}$ in $V$ and $g_{1}$, $\ldots, g_{k}$ in $R, A(V, R)$ is the algebra given by generators $x_{1}, \ldots, x_{n}$ and the relations $g_{1}, \ldots, g_{k}\left(g_{j}\right.$ are linear combinations of monomials $x_{i} x_{s}$ for $1 \leq i, s \leq n)$. Since each quadratic algebra $A$ is degree graded,

\section{We can consider its Hilbert series}

$$
H_{A}(t)=\sum_{j=0}^{\infty} \operatorname{dim}_{\mathrm{k}} A_{j} t^{j}
$$

Quadratic algebras whose Hilbert series is the same as for the algebra $\mathbb{k}\left[x_{1}, \ldots, x_{n}\right]$ of commutative Polynomials play a particularly important role in physics. We say that $A$ is a PHS (for' polynomial Hilbert series) if

$$
H_{A}(\mathrm{t})=\mathrm{H}_{\mathbb{k}\left[\mathrm{x}_{1}, \ldots, \mathrm{x}_{\mathrm{n}}\right]}(\mathrm{t})=(1-\mathrm{t})^{-\mathrm{n}}
$$

Following the notation from the Polishchuk, Positselski book [8], we say that a quadratic algebra $A=A(V, R)$ is a $P B W$-algebra (Poincare, Birkhoff, Witt) if there are bases $x_{1}, \ldots, x_{n}$ and $g_{1}, \ldots, g_{m}$ in $V$ and $R$ respectively such that with respect to some compatible with multiplication well-ordering on the monomials in $x_{1}, \ldots, x_{n}, g_{1}, \ldots, g_{m}$ is a (non-commutative) Grobner basis of the ideal $I A$ generated by $R$. In this case, $x_{1}, \ldots, x_{n}$ is called a $P B W$-basis of $A$, while $g_{1}, \ldots, g_{m}$ are called the $P B W$-generators of $I A$. In order to avoid confusion, we would like to stress from the start that Odesskii [9] as well as some other authors use the term PBW-algebra for what we have already dubbed PHS. Since we deal with both concepts, we could not possibly call them the same and we opted to follow the notation from [8].

Another concept playing an important role in this paper is Koszulity. For a quadratic algebra $A=A(V, R)$, the augmentation map $A \rightarrow \mathbb{k}$ equips $\mathbb{k}$ with the structure of a commutative graded $A$-bi module. The algebra $A$ is called Koszul if $\mathbb{k}$ as a graded right $A$-module has a free resolution

*Corresponding author: Natalia lyudu, School of Mathematics, The University of Edinburgh, The King's Buildings, Mayfield Road, Edinburgh, Scotland EH9 3JZ, E-mail: niyudu@staffmail.ed.ac.uk

Received September 30, 2014; Accepted October 06, 2014; Published October 06, 2014

Citation: lyudu N, Shkarin S (2014) Constructive Approach to Three Dimensional Sklyanin Algebras. J Generalized Lie Theory Appl 8: e101. doi: 10.4172/17364337.1000e101

Copyright: () 2014 lyudu N et al. This is an open-access article distributed under the terms of the Creative Commons Attribution License, which permits unrestricted use, distribution, and reproduction in any medium, provided the original author and source are credited. 
Citation: Iyudu N, Shkarin S (2014) Constructive Approach to Three Dimensional Sklyanin Algebras. J Generalized Lie Theory Appl 8: e101. doi: 10.4172/1736-4337.1000e101

Page 2 of 2

$\ldots \rightarrow M \rightarrow \ldots \rightarrow M_{1} \rightarrow A \rightarrow \mathbb{k} \rightarrow 0$ with the second last arrow being the augmentation map and with each $M_{m}$ generated in degree $m$. The last property is the same as the condition that the matrices of the maps $M_{m} \rightarrow$ $M_{m-1}$ in the last sequence with respect to some free bases consist of elements of $V$ (=are homogeneous of degree 1$)$.

For $a, s \in \mathbb{k}$ a Sklyanin algebra $S^{a, s}$ with 3 generators is the quadratic algebra over $\mathbb{k}$ with generators $x, y, z$ given by 3 relations

$y z-a z y-s x x=0, z x-a x z-s y y=0, x-a y x-s z z=0$.

Odesskii [9] proved that in the case $\mathbb{k}=\mathbb{C}$, a generic Sklyanin algebra is a PHS. That is, $H_{s^{a, s}}(t)=\sum^{\infty} \frac{(j+2)(\mathrm{j}+1)}{2} t^{j}$ for generic $(a, s) \in \mathbb{C}^{2}$

By generic he means outside the union of countably many proper algebraic varieties in $\mathbb{C}^{2}$. In particular, the equality above holds for almost all $(a, s) \in \mathbb{C}^{2}$ with respect to the Lebesgue measure. Polishchuk and Positselski [8] showed in the same setting and with the same understanding of the word generic that for generic $(a, s) \in \mathbb{C}^{2}$ , the algebra $S$ is Koszul but is not a PBW-algebra. The same results contained in Artin, Shelter paper [10]. The rather tricky arguments of Odesskii are based upon using a geometric interpretation of $S^{a, s}$ to show the existence of a degree 3 central element in $S^{a, s}$, which generically happens to be nota zero divisor, the arguments of Polishchuk and Positselski are essentially algebro-geometric. The drawback of this kind of results is that they are of no help, if we wish to determine whether $S^{a, s}$ is a PHS or is Koszul for any specific choice of the parameters. In the present paper we address this issue. Despite the fact that Odesskii $[9,11]$ argues that classical combinatorial techniques are in adequate for determining the Hilbert series of Sklyanin algebras, we use these techniques and they turn out to be quite helpful. Recently Sokolov [12] asked whether there exist a constructive way to determine, for which paprameters (generalized) Sklyanin algebras are PHS. This motivates us to look for constructive proofs of known results on Koszulity, PBW and PHS properties of 3-dimensional Sklyanin algebras, due to Artin, Tate, Van Den Bergh, which do not use the power of algebraic geometry. We prove the following, and our proof based entirely on Grobner basis computations, relations between Koszul algebras and their Hilbert series, and certain other arguments of combinatorial nature. This approach is substantially different from the proofs in Artin, Tate, Van Den Bergh papers [13,14], for example, they get the fact that Sklyanin algebras are PHS as a byproduct of Koszulity. We do it the other way around, we find the Hilbert series first, and then use it to prove Koszulity [15].

Theorem 0.1: The algebra $S^{a, s}$ is not a PHS if and only if and only if either $a=s=0$ or $a^{3}=s^{3}=-1$. Furthermore, the algebra $S^{a, s}$ is Koszul for any choice of $a$ and $s$. To complete the picture we determine which of these algebras are PBW.

Theorem 0.2: The algebra $S^{a, s}$ is $P B W$ if and only if either $s=0$ or $a^{3}=s^{3}=-1$ or $(1-a)^{3}=s^{3}$ and the equation $t^{2}+t+1=0$ has a solution in $\mathbb{k}$ Note that the condition of solvability of the quadratic equation above is automatically satisfied if $\mathbb{k}$ is algebraically closed or if $\mathbb{k}$ has characteristic 3 . On the other hand, if $\mathbb{k}=\mathbb{R}$ the third case is empty.

We also study the case of generalized Sklyanin algebras, namely we show that if instead of keeping coefficients in the relations to be triples of the same numbers $p, q$, $r$, we allow them to be all different, the situation changes dramatically. For instance, we show that generically such algebras are finite-dimensional and non-Koszul.

\section{Acknowledgements}

We are grateful to IHES and MPIM for hospitality, support, and excellent research atmosphere. This work is funded by the ERC grant 320974 and the ESC Grant N9038.

\section{References}

1. Kontsevich Maxim (1993) Formal (non) commutative symplectic geometry. The Gel'fand Mathematical Seminars, Birkhuser Boston, Boston, MA.

2. Dubois Violette M (2007) Multilinear forms and graded algebras. J. Algebra 317: 198-225.

3. Bocklandt R, Schedler T, Wemyss M (2010) Super potentials and higher order derivations. J. Pure Appl Algebra 214: 1501-1522.

4. Ginzburg V (2007) Calabi-Yau algebras, Cornell University Library 3: 1-79.

5. Estanislao H, Andrea S (2012) Hochschild and cyclic homology of Yang-Mills algebras. J Reine Angew Math 665: 73-156.

6. Ginzburg V (2007) Calabi-Yau algebras, Cornell University Library 3: 1-79.

7. Sklyanin EK (1983) Some algebraic structures connected with the Yang-Baxter equation. Representations of quantum algebra. Functional Analysis and Its Applications 17: 273-284

8. Polishchuk A, Positselski L (2005) Quadratic algebras, University Lecture Series 37: 596

9. Odesskii AV (2002) Elliptic algebras. Russian Math Surv 57: 1127.

10. Artin M, Shelter W (1987) Graded algebras of global dimension 3. Adv in Math 66: 171-216.

11. Odesskii AV, Feigin BL (1989) Sklyanin's elliptic algebras. Functional Analysis and Its Applications 23: 207-214

12. V.Sokolov (2014) private communication, IHES.

13. Artin M, Tate J, Van den Bergh M (1991) Modules over regular algebras of dimension 3.Invent math 106: 335-388.

14. Artin M, Tate J, Vanden Bergh M (1990) Some algebras associated to auto morphism of elliptic curves. The Grothendieck Festschrift, Progr. Math 86,Birkhuser Boston, Boston, MA, Vol. I, 3385

15. Walton C (2012) Representation theory of three-dimensional Sklyanin algebras. Nuclear Phys. B 860: 167-185. 